\title{
Feature extraction of ECG signals for early detection of heart arrhythmia
}

\author{
K. Akilandeswari ${ }^{1}$, R. Sathya ${ }^{2}$ \\ Department of Computer Science, Government Arts College (Autonomous), Salem, India ${ }^{1,2}$
}

\begin{abstract}
In today's world, irrespective of age, cardiac arrhythmia (heart abnormalities) is common among people, which must be detected and treated at the very earliest stage. Electrocardiograph (ECG) plays a vital role in detecting various cardiac arrhythmias. ECG waveform provides entire information about the electrical activity of the heart. In this paper, we are mainly focusing on the primary attributes P, Q, R, S, T, their segments and intervals of ECG Signals. Walsh-Hadamard transformation (WHT) and Fast Fourier Transformation (FFT) are performed to extract the required features from ECG signal.
\end{abstract}

Keywords: ECG signals, Feature Extraction, FFT, WHT, and Classification.

\section{INTRODUCTION}

ECG is one of the simplest and best cardiac investigations available. It provides a huge amount of useful information and remains as an essential part of diagnosis and treatment $\mathrm{X}$ for cardiac patients. An ECG is simply a representation of the electrical activity of the heart muscle as it changes over time, usually printed on paper for easier analysis [1]. Cardiac muscle contracts in response to electrical depolarization of the muscle cells. Sum of this electrical activity, when amplified and recorded for just a few seconds that we know as an ECG [1]. ECG signals must be pre-processed before performing cardiac arrhythmia classification. This pre-processing of ECG signals improves the accuracy in extraction of P-wave, QRScomplex, T-wave, their segments and intervals. These attributes form a single cardiac cycle. The features Pwave, QRS-complex, T-wave, PR-interval, RR-interval, ST-interval, QT-interval, ST-segment, and PR-segment from ECG signal are used to find the abnormal behaviour of a cardiac cycle. This pre-processing task eventually leads to an accurate classification of cardiac arrhythmia. Pre-processing is done because of noises in the ECG signal which leads to false detection [2]. Noise sources are Baseline wander, Power line interference, Muscle noise, Instability of electrode-skin contact, Instrumentation and electrosurgical noise.

\section{LITERATURE SURVEY}

Denoising of ECG signal using undecimated wavelet transform was proposed by Baliram S. Gayal1, F.I. Shaikh [3] where the authors suggested that raw ECG signals were decomposed with UWT and shrinkage operation was performed to eliminate noise from the ECG signal. Semisoft thresholding operators along with traditional hard and soft thresholding operators were used in shrinkage step. The suitability of different wavelets was verified for denoising of ECG signal. The unique characteristics of the UWT transform comparing with the DWT were Invariant Translation Characteristic, better Capacity to reduce noise and better peak detection.
ECG QRS Complex Detection Using Slope Vector Waveform (SVW) Algorithm was proposed by Xiaomin $\mathrm{Xu}$ and Ying Liu [4] for finding RR-interval and QRS complex of ECG signal. They implemented this technique for the determination of better signal to noise ratio, nonlinear amplification and also used differentiator to detect the desired slope vectors for features of ECG wave.

Mathematical Morphology Based ECG Feature Extraction for the Purpose of Heartbeat Classification was proposed by Tadejko and Rakowski [5] for the detection of abnormal heart beat. They used Kohonen self-organizing maps (SOM) for finding the ECG wave features from the ECG signal. Correlation analysis for abnormal ECG signal features extraction was proposed by A. B. Ramli, and P. A. Ahmad [6] for finding important features of ECG signal by using Cross-Correlation signal analysis technique.

A rough set based inference engine for ECG classification was proposed by S. Mitra, M. Mitra, and B. B. Chaudhuri [7], where they used three stage detection of heart diseases. First technique was the SWT for de-noising ECG signal, morphological features extraction of ECG signal as the second technique and multilayer neural network for classification as the third technique. The use of Levenberg-Marquardt (LM) algorithm achieved the best overall detection accuracy.

A patient adapting heart beat classifier using ECG morphology and heartbeat interval features was proposed by F. de Chazal and R. B. Reilly [8] for the recognition of premature ventricular contraction (PVC) from the normal beats and other heart diseases with the help of de-noising module, feature extraction module and classifier module. Stationary wavelet transform (SWT) were used for reducing the effect of noise in heartbeat. Combination of the morphological-based features and timing intervalbased features were used in ECG signal feature extraction. 
ECG feature extraction using optimal mother wavelet was • proposed by B. Castro, D. Kogan, and A. B. Geva [9] where the author suggested the wavelet transform technique for feature extraction and also proposed an algorithm for feature extraction of ECG signal. They used soft or hard threshold for de-noising the ECG signal. ECG signal feature was divided into coefficient vector by optimal wavelet transformation. They chose mother wavelet transformation of orthogonal and bi-orthogonal wavelet filter bank by means of the best correlation with the ECG signal was developed. After the analysis of ECG signal, coefficients are divided into QRS complex, $\mathrm{T}$ wave, and $\mathrm{P}$ wave then sum to obtain feature extraction.

Wavelet based QRS detection in ECG using MATLAB was proposed by K.V.L. Narayana and A. Bhujanga Rao [10] where they suggested that Daubechies wavelet was chosen because it picks up minute details that were missed by other wavelet algorithm.

\section{III.METHODOLOGY}

The following Fig. 1 shows how the features are extracted from the ECG signal:

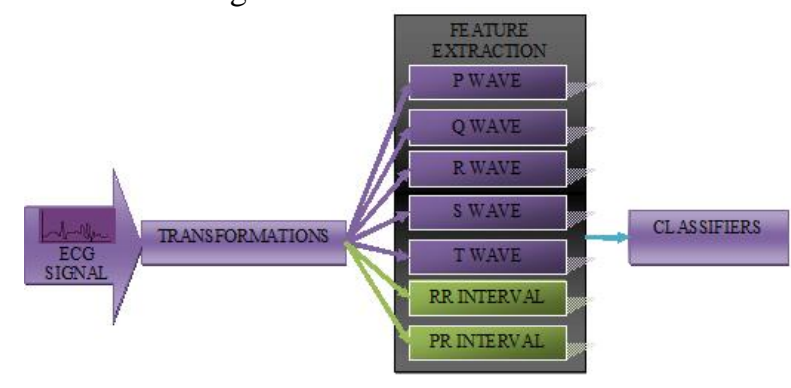

Fig. 1 ECG Signal Feature Extraction

\section{A. ECG theory}

Electrocardiography is used to interpret the electrical activity of the human heart. ECG varies from person to person based on their heart conditions. Electrodes are placed on the surface of the skin which further records the electrical activity of the heart over a particular period of time. ECG signals are non-stationary waves [11]. Normally an ECG cycle comprises of P-wave, QRS complex, T-wave which looks like Fig. 2.

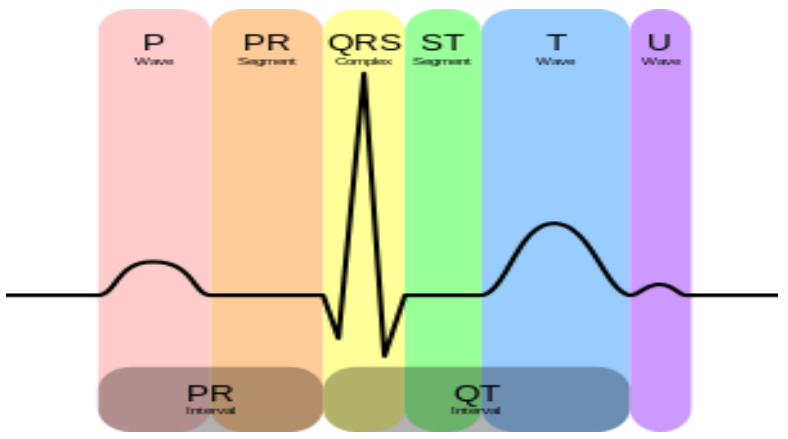

Fig. 2 Normal cycle of Heart Beat [GOOGLE SOURCE]

Description of a cardiac cycle:

- $\quad$ P wave - Atrial Depolarization (contraction).
PR segment - Indicative of the delay in the AV node which is measured from offset of $\mathrm{P}$-wave to onset of Q-wave.

- $\quad$ PR interval - Refers to all electrical activity in the heart before the impulse reaches the ventricles.

- $\quad$ Q wave - First negative (down ward) deflection after the $\mathrm{P}$ wave but before the $\mathrm{R}$ wave.

- $\quad$ wave - First positive (upward) deflection following the $\mathrm{P}$ wave.

- $\quad$ S wave - First negative (down ward) deflection after the $\mathrm{R}$ wave.

- QRS complex - Signifies ventricular depolarization.

- T wave - Indicates ventricular repolarization (relaxation).

Feature Extraction for ECG signals is done by dimensionality reduction in which the most vital features of ECG signal are represented as compact feature vector [12]. The features were extracted through segmenting signals based on location of the peaks.

\section{B. Dataset}

MIT BIH arrhythmia dataset is used for extracting the features of ECG signal. It contains 4000 long-term Holter recordings that were obtained by the Beth Israel Hospital Arrhythmia Laboratory. It contains 48 inpatient records [13].

\section{Transformations}

Transform is a mathematical tool which is used to move from time domain to frequency domain. Transforms change the representation of signal by projecting it onto a set of basic functions but do not change the signal information content. In this paper we are providing the comparative study of the following two transformations:

\section{1) Walsh-Hadamard Transformation}

The Walsh-Hadamard transform (WHT) is a suboptimal, non-sinusoidal, orthogonal transformation that decomposes a signal into a set of orthogonal, rectangular waveforms called Walsh functions. The amplitude of Walsh (or Hadamard) functions has only two values, +1 or -1. An important characteristic of Walsh functions is sequence which is determined from the number of zerocrossings per unit time interval. Every Walsh function has a unique sequence value [6]. WHT based transformed ECG signal is shown in Fig. 4.

\section{2)}

\section{Fast Fourier Transformation}

Fast Fourier transformation is an efficient transform technique which is based on the decomposition of signals to smaller transforms and combining them to get total transform. N point's complex signal will be decomposed into $\mathrm{N}$ individual points. Each of the complex ECG signals composed of real part and imaginary part. Low frequencies are removed from ECG signal via Fast Fourier transformation. Noises are removed via Inverse Fast Fourier transformation. FFT based transformed ECG signal is shown in Fig. 3. 


\section{RESULTS AND DISCUSSIONS}

The input signals from the dataset were pre-processed by removing null values and transformed using Fast Fourier Transformation and Walsh-Hadamard Transformation, which were found to be noise free. The signals before and after transformations are shown in the following figures. These results were obtained using Matlab and extracted features are suitable for detecting patient records with heart arrhythmia. The produced excel file could be used for classification and finding different abnormalities.

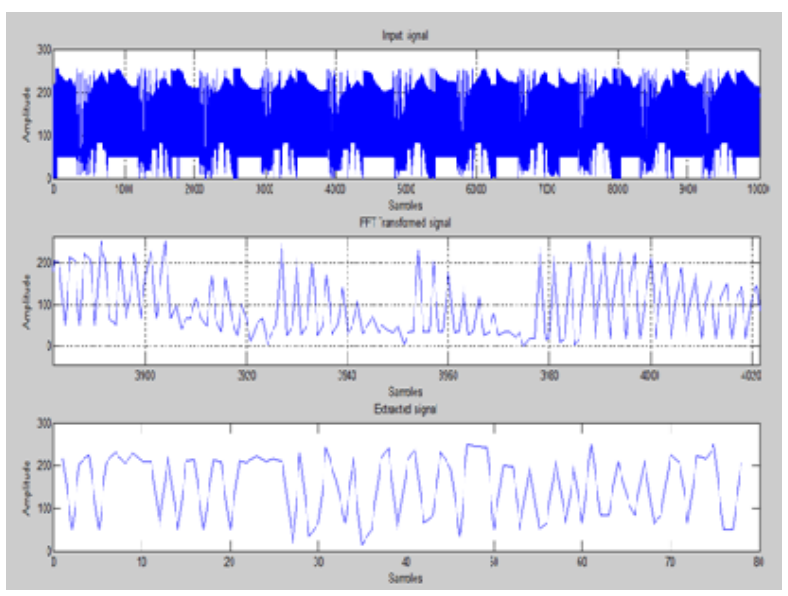

Fig. 3 Transformation using Fast Fourier Transformation.

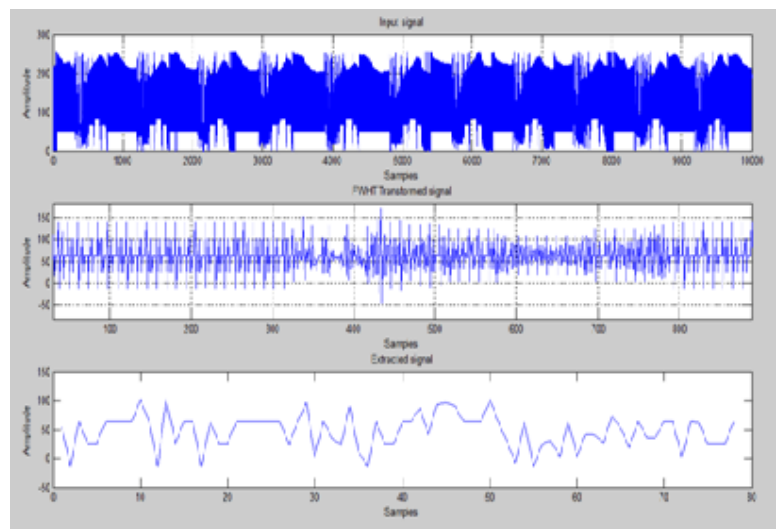

Fig. 4 Transformation using Walsh-Hadamard Transformation

\section{REFERENCES}

[1] http://www.southsudanmedicaljournal.com/archive/may-2010/howto-read-an-electrocardiogram-ecg.-part-one-basic-principles-of-theecg.-the-normal-ecg.html.

[2] http://en.wikipedia.org/wiki/Electrocardiography.

[3] Baliram S. Gayal1, F.I. Shaikh, Denoising of ECG signal using undecimated wavelet transform, International Journal of Advanced Research in Electrical, Electronics and Instrumentation Engineering Vol. 3, Issue 1, January 2014.

[4] Xiaomin Xu, and Ying Liu, 2004. ECG QRS Complex Detection Using Slope Vector Waveform (SVW) Algorithm, Proceedings of the 26th Annual International Conference of the IEEE EMBS, pp. 3597-3600.

[5] P. Tadejko, and W. Rakowski, 2007. Mathematical Morphology Based ECG Feature Extraction for the Purpose of Heartbeat Classification, 6th International Conference on Computer Information Systems and Industrial Management Applications, CISIM '07, pp. 322-327.

Copyright to IJARCCE 